\title{
COVID-19 Patient Management in Intensive Care
}

\author{
(D) Şule Vatansever, (D) Esra Akdaş Tekin, (D) Fethi Gültop, (D) Fulya Uğurlu, (D) Tarkan Mıngır, (D) Onur Okur, (D) Cengiz Polat, \\ (D) Namigar Turgut \\ University of Health Seciences Turkey, Prof. Dr. Cemil Taşçıoğlu City Hospital, Clinic of Anesthesiology and Reanimation, Istanbul, Turkey
}

\section{Abstract}

The World Health Organization (WHO) declared the coronavirus outbreak as an international public health emergency (pandemic) on January 31, 2020. In line with WHO recommendations, in order to guide the Coronaviruse Disease-2019 (COVID-19) agent, transmission routes, case definitions, diagnosis and treatment methods, strategy and application methods, Turkish Ministry of Health, General Directorate of Public Health has prepared guidelines. Management of patients with COVID-19 includes epidemiological risk assessment and patient isolation; general and optimized supportive care, symptomatic treatment, respiratory support, nutritional support, psychological intervention etc. for infection prevention and control. The prognosis of patients depends on the severity of the disease, the age of the patient, their underlying diseases and their general medical condition. Patients with severe COVID-19 should be treated in the critical care unit.

Keywords: COVID-19, intensive care, supportive therapy

\section{INTRODUCTION}

In December 2019, a new coronavirus was detected in Wuhan, China, which was widespread, highly contagious and caused a high rate of acute respiratory disease. The World Health Organization (WHO) declared the coronavirus outbreak to be an international public health emergency (pandemic) on January 31, 2020.

In line with the recommendations of WHO, guidelines were prepared in order to guide about the Coronaviruse Disease-2019 (COVID-19) agent, transmission routes, case definitions, diagnosis and treatment methods, strategy and implementation methods, especially by the Ministry of Health, General Directorate of Public Health [COVID-19 Severe Acute Respiratory syndromecoronavirus-2 (SARS-CoV-2) Infection Guide 14.04.2020] (1).

Management of patients with COVID-19 includes epidemiological risk assessment and patient isolation; general and optimized supportive care, symptomatic treatment, respiratory support, nutritional support, and psychological intervention, etc for infection prevention and control (2).

COVID-19 may present with severe pneumonia, Acute Respiratory Distress syndrome (ARDS), sepsis, septic shock, myocarditis, arrhythmia and cardiogenic shock, and multiple organ failure.

Respiratory failure is often hypoxemic respiratory failure, less commonly in the form of hypercapnic respiratory failure. The prognosis of patients depends on the severity of the disease, the patient's age, underlying diseases, and general medical condition. Severe patients with COVID-19 should be treated in a critical care unit [intensive care unit (ICU)].

\section{COVID-19 Management in the Intensive Care Unit of the Ministry of Health Prof. Dr. Cemil Taşcıoğlu City Hospital and Okmeydani Training and Research Hospital}

\section{Infection Control and Tests}

During aerosol-generating procedures (Endotracheal intubation, open aspiration, nebulized treatments, manual ventilation before 
intubation, taking the patient into the prone position, leaving the patient from the ventilator, non-invasive positive pressure ventilation, tracheostomy and cardiopulmonary resuscitation), healthcare workers wear gloves, overalls, safety glasses or face shield, N95 mask, FFP2 or equivalent. Endotracheal intubation, if available, is recommended to be performed with videolaryngoscopy instead of direct laryngoscopy by a healthcare professional with the most experience in airway management to minimize the number of attempts and the risk of contamination.

In order to protect healthcare professionals and other patients in the hospital by preventing the spread of infectious airborne pathogens from room to room or open areas in our ICU, the entire 48-bed ICU, each room of which is an isolated single room, has been turned into a negative pressure room. Contamination between the rooms has been prevented, and the risk of infection in the patients and employees outside the room has been reduced when aerosol-forming processes are performed in the room such as tracheal intubation, bronchoscopy and a noninvasive ventilation. In addition, unnecessary staff has not been allowed in the patient room.

\section{Support Therapy}

The clinical spectrum of COVID-19 ranges from mild illness to ARDS with a high risk of mortality, with early markers of disease severity not yet fully established. Current evidence suggests that patients with advanced age, pre-existing comorbidity or dyspnea should be closely monitored, especially 1 to 2 weeks after symptom onset. More studies are needed to understand the incidence and consequences of COVID-19 ARDS, and to conduct critical care management and resource planning (3).

\section{Hemodynamic Support}

ARDS, severe pneumonia, sepsis, and septic shock are the lifethreatening forms of SARS-CoV-2 infection and are indicative for treatment in the ICU (4). The prevalence of shock in adult COVID-19 patients is highly variable (1-35\%) depending on the patient population, the severity of the disease, and the definition of shock.

In the meta-analysis of 660 articles (1/1/2020-2/23/2020) by Rodriguez-Morales et al. (5), 20.3\% of the patients needed intensive care, and $32.8 \%$ of these patients were admitted to ICU in ARDS and $6.2 \%$ in shock. Average hospital mortality rate was $13.9 \%$. In a study conducted with 44.415 patients, 2087 (5\%) patients were defined as critical patients with other organ failure, including severe hypoxemia and shock (6). It is probably more common in hospitalized patients and can reach 20-35\% in patients in ICUs (7). It should be kept in mind that patients may have myocarditis and related arrhythmia and cardiogenic shock. Cardiac damage was found between $7 \%$ and $23 \%$ of patients with COVID-19 in Wuhan (7). They suggested that advanced age, low lymphocyte count, high D-dimer level were risk factors for possible cardiac damage.

Between 20 March and 15 May 2020, 1910 patients were admitted to our hospital, $9.1 \%$ of them (175 patients) were admitted to ICU and advanced hemodynamic support was required.

\section{Fluid Therapy}

In order to evaluate the fluid response in patients with COVID-19 and shock, it is recommended to use dynamic parameters (body temperature, capillary refill time and serum lactate levels) instead of static parameters. In acute resuscitation, conservative approach rather than liberal fluid strategy, using buffered balanced crystalloid instead of colloid is appropriate. Gelatins and dextrans should not be used. It is recommended to administer $30 \mathrm{~mL} / \mathrm{kg}$ isotonic crystalloid fluid (saline or ringer's lactate) in the first hour, however, fluid therapy should be carried out carefully and hypervolemia should be avoided in patients with ARDS. Albumin should not be used routinely for initial resuscitation. In patients with COVID-19, despite fluid resuscitation, vasopressor support should be given in the presence of shock or in very deep hypotension, with an average arterial pressure of $65 \mathrm{mmHg}$ and the first choice is the vasoactive agent norepinephrine. When not available, it is appropriate to use vasopressin or epinephrine as the first choice instead of other agents. Subsequent treatment is determined by the patient's cardiac output and fluid responsiveness. Dopamine should not be used. In case of persistence of cardiac dysfunction despite adequate fluid resuscitation and norepinephrine and ongoing signs of hypoperfusion, it is appropriate to add dobutamine instead of increasing the norepinephrine dose. Lactate monitoring should be done. In patients with refractory shock, daily intravenous $200 \mathrm{mg}$ hydrocortisone can be used as a low-dose infusion or as an intermittent dose instead of giving corticosteroid therapy. Our practice in the clinic has been in this direction.

\section{Respiratory Failure Management}

In SARS-CoV-2 infection, acute hypoxemic respiratory failure requiring mechanicalventilation developsdueto manyunderlying mechanisms such as pulmonary edema, hemoglobinopathy, vascular occlusion and ventilation/perfusion mismatch (7). If peripheral oxygen saturation $\left(\mathrm{SpO}_{2}\right)$ is $<90 \%$, oxygen therapy should be initiated. Recent guidelines recommend a target oxygen saturation of $92-96 \%$ in adults with COVID-19 using 
supplemental oxygen as needed. If acute hypoxemic respiratory failure develops despite conventional oxygen therapy, high frequency nasal oxygen [high flow nasal cannula (HFNC), up to $60 \mathrm{~L} / \mathrm{min}$ ] can be used instead of noninvasive mechanical ventilation (NIMV). NIMV should be administered with intensive care ventilators or dual circuit ventilators. A viral/bacterial filter should be added to the inspiratory and expiratory outputs of the circuits. It should be applied with maximum personal protective equipment (PPE) in negative pressure rooms if possible, and in single rooms if possible, especially due to the risk of aerosol creation of high flow oxygen application and NIMV (8). Patient selection is important; those with moderate to severe hypoxaemia are unlikely to receive sufficient oxygen through HFNC and usually require intubation. Stable patients with mild to moderate hypoxaemia can be given oxygen via nasal cannulas, but should be carefully monitored for signs of deterioration. Patients should be evaluated for worsening of respiratory failure at close monitoring and at short intervals. In case of worsening in breathing, early intubation should be considered (Appendix 1).

Although the actual incidence of hypoxic respiratory failure in patients with COVID-19 is not known, approximately $14 \%$ of the patients will be in a serious condition that requires oxygen therapy and 5\% will need mechanical ventilation and intensive care (6). Of the patients hospitalized in our hospital, 9.1\% needed mechanical ventilation and intensive care.

Controversies against early intubation remain unresolved, largely due to lack of good evidence. Those recommending early intubation recommend avoiding the risk of self-induced lung injury. Those who oppose early intubation note the high mortality rates reported among intubated patients with COVID-19 and claim that ventilator-induced lung injury is a contributing factor. The best approach will depend on the patient. A single approach is unlikely to work in a disease as heterogeneous as COVID-19 (9).

Although the current histopathology shows widespread alveolar damage consistent with ARDS, Gattinoni et al. (9) has stated that COVID-19 pneumonia is a specific disease with distinctive phenotypes with relatively well preserved respiratory mechanics despite the severity of hypoxemia.

There are two types of patients with different pathophysiologies who are clearly distinguishable by computered tomography scan;

Type 1: Approximately $2 / 3$ of the patients who do not have ARDS and whose compliance is preserved are in this form.

Type 2: ARDS, low compliance.
Type 1 patients: In patients with high pulmonary compliance (static $>40 \mathrm{~mL} / \mathrm{cmH}_{2} \mathrm{O}$ ), positive end-expiratory pressure (PEEP) levels should be kept low, tidal volume should not exceed $6 \mathrm{~mL} /$ $\mathrm{kg}$, respiratory frequency should not exceed 20/min.

\section{Acute Respiratory Distress Syndrome}

- Respiratory distress that occurs or worsens in the last week

- Radiologically pleural effusion, collapse or nodular bilateral opacities

- Respiratory failure that cannot be explained by heart failure or volume excess

- Mild ARDS: $200<\mathrm{PaO}_{2} / \mathrm{FiO}_{2} \leq 300\left(\mathrm{PEEP} \geq 5 \mathrm{cmH}_{2} \mathrm{O}\right)$

- Moderate ARDS: $100<\mathrm{PaO}_{2} / \mathrm{FiO}_{2} \leq 200\left(\mathrm{PEEP} \geq 5 \mathrm{cmH}_{2} \mathrm{O}\right)$

- Severe ARDS: $\mathrm{PaO}_{2} / \mathrm{FiO}_{2} \leq 100\left(\mathrm{PEEP} \geq 5 \mathrm{cmH}_{2} \mathrm{O}\right)$

Since ARDS (Type 2) caused by COVID-19 is similar to ARDS due to other causes, the principles supporting ventilation should also be similar. Standard therapy should be used for severe ARDS; low tidal volume (Vt 4-8 mL/kg) instead of higher tidal volume (Vt $>8$ $\mathrm{mL} / \mathrm{kg}$ ), plateau pressures (Pplat) $<30 \mathrm{cmH}_{2} \mathrm{O}$, driving pressure (driving pressure $=$ plateau pressure - PEEP) $<14 \mathrm{cmH}_{2} \mathrm{O}$, it is recommended to use a higher PEEP strategy rather than a low PEEP strategy. In moderate-severe patients $\left(\mathrm{PaO}_{2} / \mathrm{FiO}_{2}<200\right)$, PEEP, which provides the best compliance and oxygenation without impairing hemodynamics, should be applied. In cases of $\mathrm{pH}<7.15$ and hypercapnia, tidal volumes can be increased to $8 \mathrm{~mL} / \mathrm{kg}$, and respiratory rate can be up to $30 / \mathrm{min}$. Otherwise, permissive hypercapnia may be allowed. It is sufficient to have $\mathrm{PaO}_{2}$ of $60-85 \mathrm{mmHg}$ and $\mathrm{SO}_{2}$ of $88-95 \%$ (10). Despite ventilation optimization, recruitment maneuvers can be used in case of hypoxemia. The use of neuromuscular blocking agents (NMBA) is not recommended routinely, but can be applied in the presence of ventilator incompatibility, persistent hypoxemia or hypercapnia despite sedation. Excessive sedation should be avoided or sedation should be light (Figure 1). Thrombosis and associated ischemic events are very common in this disease. Anticoagulation should be initiated when necessary and daily coagulation parameters, especially D-dimer levels should be checked.

\subsection{Prone Position}

Uncertainty about how best to optimize respiratory support has led to increased use of the awake prone position for non-intubated patients. Lying prone improves ventilation-perfusion matching and is associated with reduced mortality in patients with ARDS compared with supine positioning. The positive effects of daily 

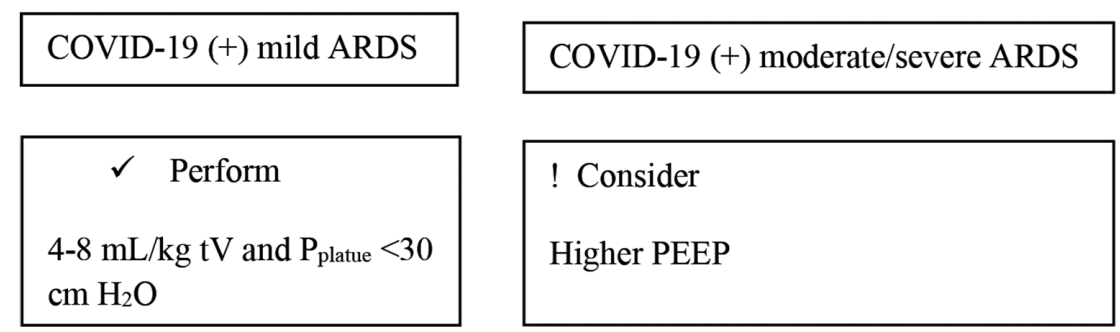

\begin{tabular}{l|} 
! Consider \\
Higher PEEP \\
\hline
\end{tabular}

\begin{tabular}{|c|}
\hline \multicolumn{1}{|c|}{ Perform } \\
$\begin{array}{l}\text { Search for presence of } \\
\text { bacterial infection }\end{array}$ \\
\hline
\end{tabular}

\section{$\checkmark \quad$ Perform}

Target $\mathrm{SPO}_{2}$ is $92-96 \%$

\begin{tabular}{|l|}
\hline Consider \\
Conservative liquid strategy
\end{tabular}

\section{! Consider}

Empiric antibiotic therapy

? Unclear
Systemic corticosteroids

\section{! Don't do it}

\section{! Consider}

Neuromuscular blocker agent boluses to facilitate ventilation targets

\section{! Consider: (If there is response to} PEEP)

\section{Conservative recruitment maneuvers}

\section{! Consider}

Prone ventilation for 12-16 hours

! Consider: High $\mathrm{P}_{\text {platue }}$ and asynchrony despite prone

Neuromuscular blocker agent infusion for 24 hours

Stepwise recruitment

! Consider

Short-term administration of systemic corticosteroids

\section{Rescue/Support treatment}

? Unclear

Antiviral agents,

hydroxychloroquine, anti-IL6

! Consider: High $\mathrm{P}_{\text {platue }}$ and asynchrony despite prone

Neuromuscular blocker agent infusion for 24 hours

! Consider

Prone ventilation for 12-16 hours

! Consider

Prone ventilation for 12-16

hours

! Consider: Stop if there is no rapid response

Nitric oxide inhalation

! Consider: See the ECMO criteria

$\mathrm{V}-\mathrm{V}$ ECMO or send to an ECMO center

\section{? Unclear}

Antiviral agents, hydroxychloroquine, anti-IL6

Figure 1. Patients with mild/moderate/severe COVID-19 and Rescue/Support therapy (10) 
prone position application on hypoxia have been demonstrated in non-intubated patients with pulmonary involvement. For type 2 patients, the prone position can be used as long-term therapy (for 12-16 hours) as with any severe form of ARDS. However, in type 1 patients, prone positioning should be considered as a rescue maneuver to facilitate the redistribution of pulmonary blood flow rather than opening up collapsed areas. Prolonged prone positioning/supine cycles provide little benefit in patients with high lung compliance and result in high levels of stress and fatigue in staff (9). While it is appropriate to use intermittent boluses of NMBA instead of continuous infusion of NMBA when necessary to facilitate protective lung ventilation; in the presence of ventilator desynchronization, ongoing deep sedation, prone ventilation, or continuous high plateau pressures, continuous NMBA infusion up to 48 hours may be required (10). In the ICUs of our hospital, prone position was provided in all patients with $\mathrm{P} / \mathrm{F}<150$ and the duration was programmed to be $12-16$ hours according to the response, and even we had applications that lasted 24 hours in some patients.

Although the ventilation is optimized by using rescue therapies and prone positioning in adults with COVID-19 who are mechanically ventilated; in case of refractory hypoxemia, it is recommended to perform venovenous extra-corporeal membrane oxygenation (ECMO) or refer the patient to an ECMO center. ECMO should only be considered in carefully selected patients with COVID-19 and severe ARDS (Figure 2, our ECMO application).

\subsection{Application of Tracheostomy}

Since tracheostomy is one of the processes that create aerosol, care should be taken in its timing. The general approach is to

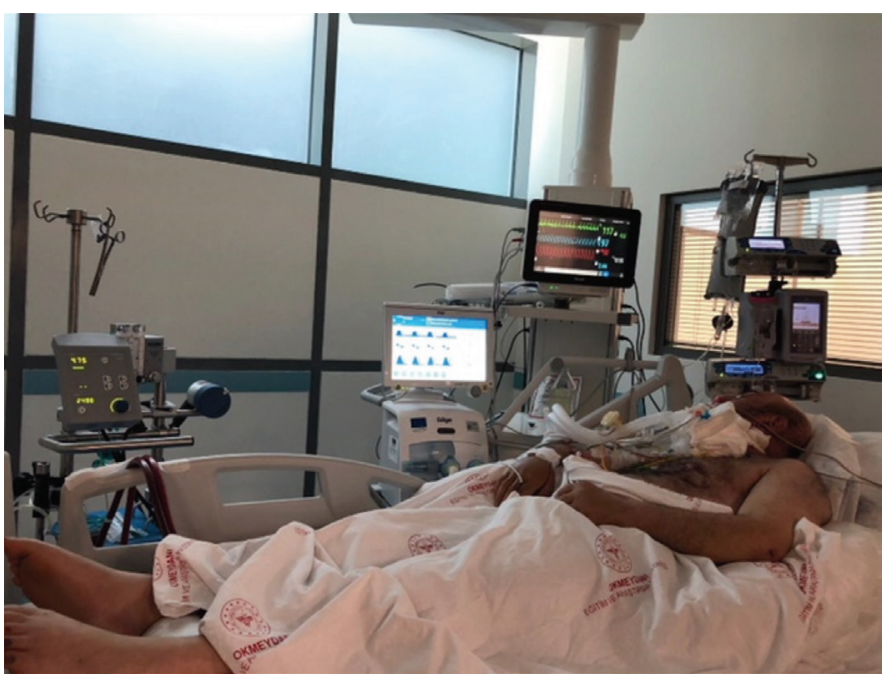

Figure 2. ECMO application

ECMO: Extra-corporeal membrane oxygenation avoid tracheostomy before the 14th day of intubation. Some authors recommend not to make a tracheostomy decision before the 21st day. These applications are also aimed at reducing the patient's viral load. Repeating the polymerase chain reaction (PCR) or antibody test before tracheostomy and a negative result are recommended by some authors, but repeating the PCR test before tracheostomy is not an accepted practice (1). Maximum PPE (N95/FFP3 mask, goggles/face protection, liquid impermeable surgical gown/overalls, double-layer gloves and, if possible, powered air-purifying respirator) should be used for healthcare worker safety. Tracheostomy should be performed by the most experienced physician. Tracheostomy should be performed in a negative pressure operating room or a negative pressure patient room. The choice of percutaneous or surgical tracheostomy method should be decided based on hospital resources and experience. The general approach is to prefer surgical tracheostomy (Appendix 2. Tracheostomy protocol. Our percutaneous tracheostomy applications in the clinic; Figures 3a, 3b, 3c, 3d).

\section{Medical Treatment of COVID-19}

\section{Anti-cytokine and Anti-inflammatory Treatments}

Tocilizumab, which blocks interleukin (IL)-6, has been reported to have a positive effect on COVID-19 related Macrophage Activation syndrome. In our unit, tocilizumab treatment is preferred in our patients under the guidance of infectious diseases clinic

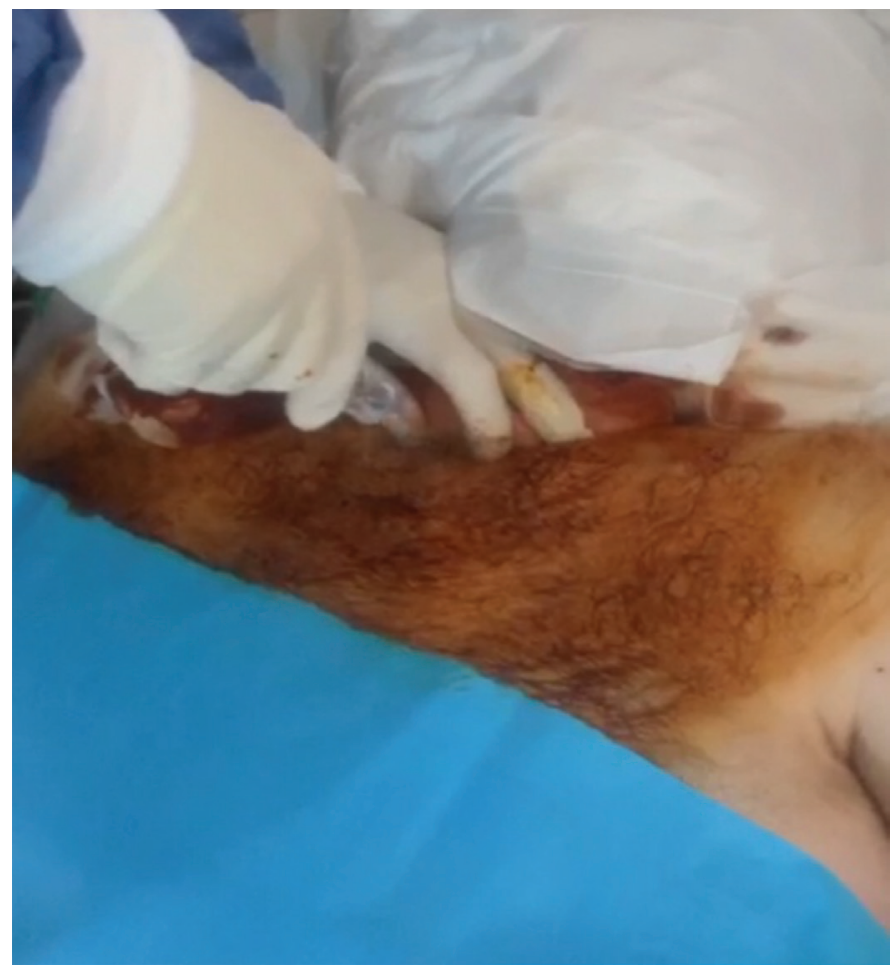

Figure 3a. Percutaneous tracheostomy; puncture 


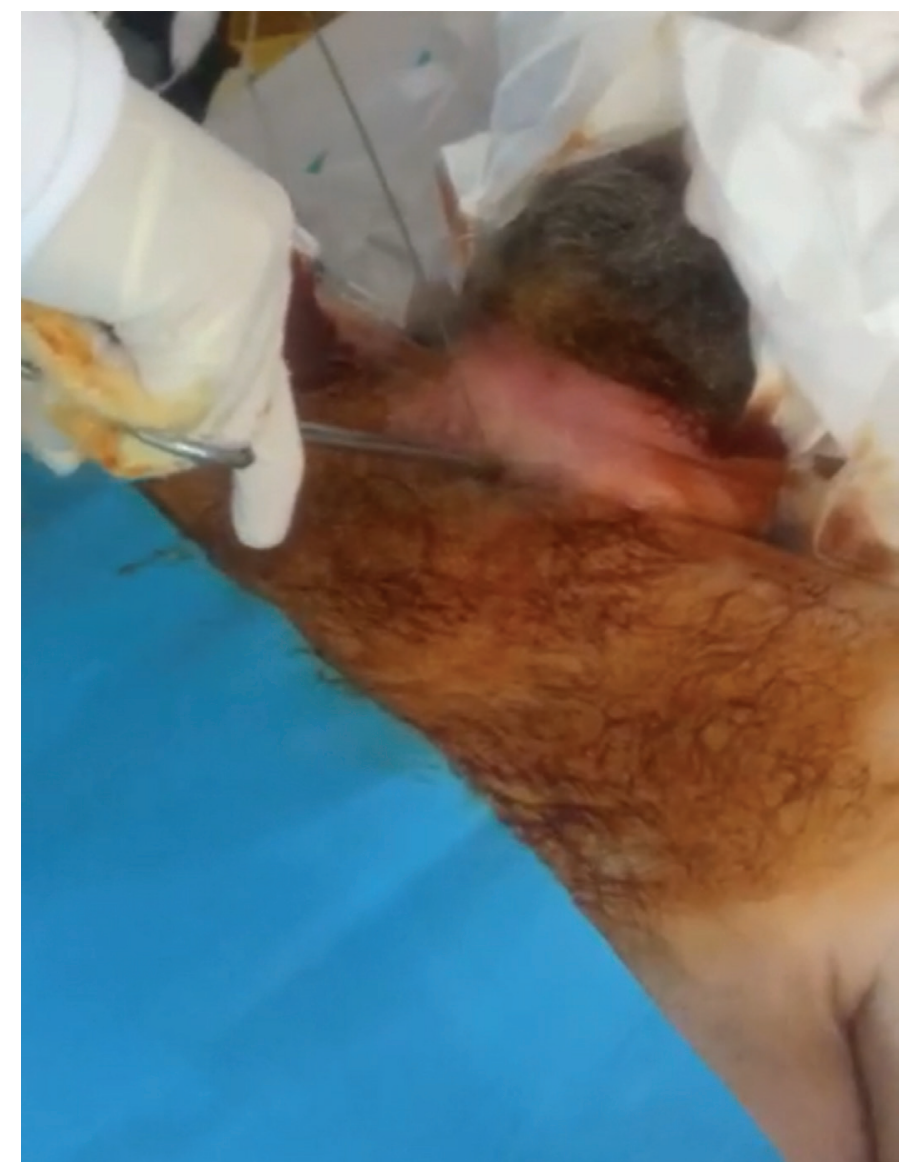

Figure 3b. Percutaneous tracheostomy; dilatation

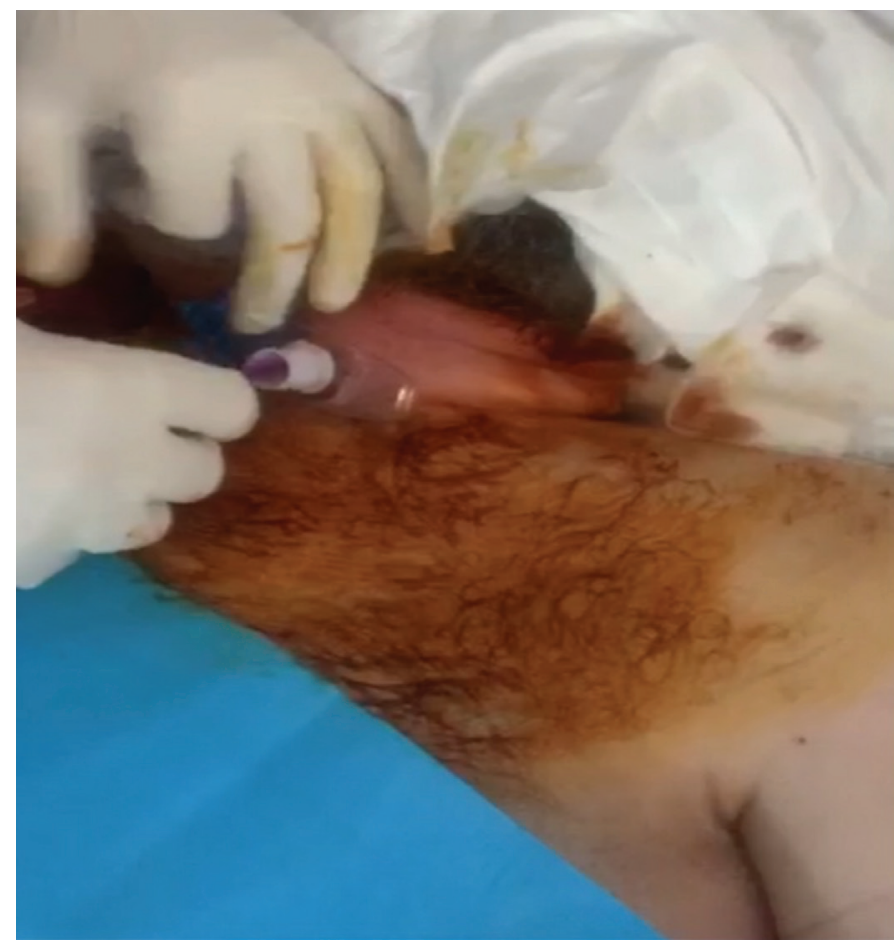

Figure 3c. Percutaneous tracheostomy; insertion of tracheostomy cannula when necessary. Excessive immune response to infection with the SARS-CoV-2 virus has been blamed for respiratory distress and multiorgan failure, referred to as "cytokine storm" in some patients. High levels of IL-6 and other cytokines have been shown.

In a study conducted with 21 patients with severe COVID-19 symptoms who were given IL-6 blocker tocilizumab; fever and elevated C-reactive protein (CRP) levels improved and levels of IL-6 and other proinflammatory cytokines decreased within 24 hours of tocilizumab therapy. Additional oxygen use decreased in 15 patients, and oxygen saturation levels stabilized in all patients. Two patients were removed from the mechanical ventilator (11). Our practices in our clinic have also been in this direction. Tocilizumab is administered at a dose of $8 \mathrm{mg} /$ $\mathrm{kg}$ (maximum $800 \mathrm{mg}$ ). It can be administered as a single dose of $400 \mathrm{mg}$ or $800 \mathrm{mg}$ intravenously, depending on the severity of the patient's symptoms. When the first dose is $400 \mathrm{mg}$, taking into account the changes in clinical and laboratory findings, a dose of 200-400 mg can be repeated within 12-24 hours (Appendix 3. ICU COVID-19 Treatment algorithm). Tocilizumab should not be used in the presence of pregnancy, neutropenia $\left(<500 / \mathrm{mm}^{3}\right)$, active tuberculosis, active hepatitis B or C, allergy, and hypersensitivity. Liver functions and thrombocyte count should be monitored. Patients with diverticulitis history should be monitored closely for perforation. Since CRP values may

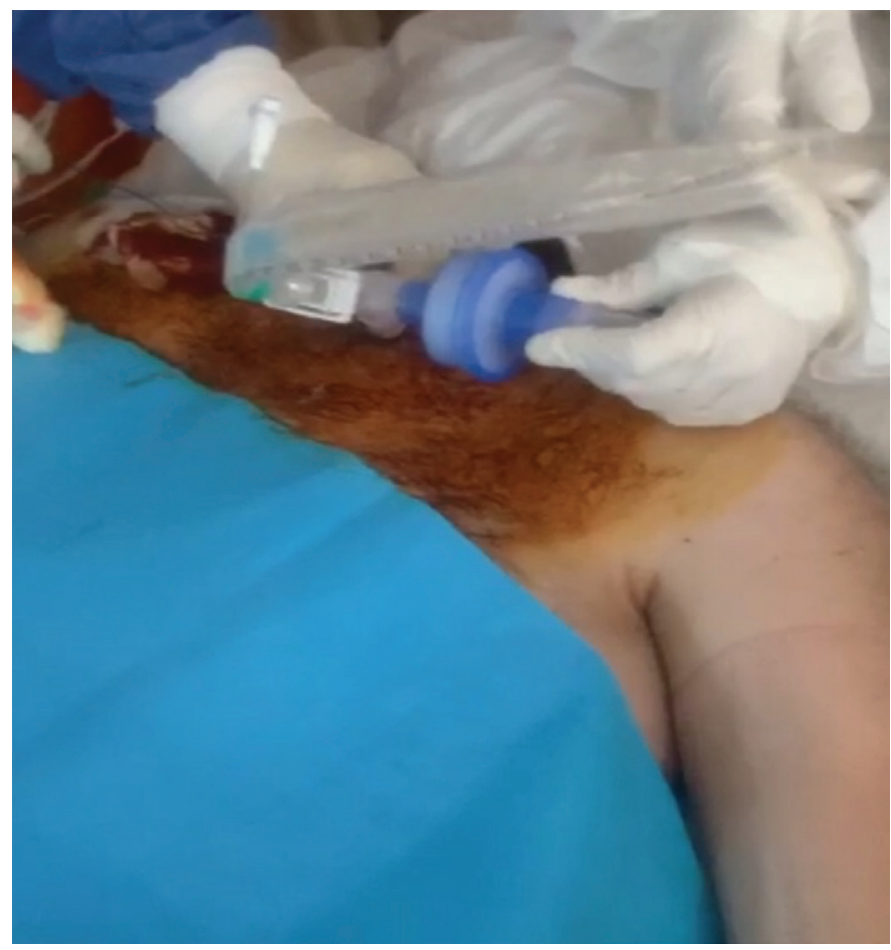

Figure 3d. Percutaneous tracheostomy; connecting to a mechanical ventilator 
decrease after tocilizumab treatment, regardless of the clinical efficacy of the drug, additional examinations (such as serum IL-6 levels) should be used in the follow-up of the acute phase response. It should be known that the decrease in ferritin values will not be rapid when there is a response to the treatment, and if the values remain high for a while, it should not be considered as treatment failure. In addition, signs of inflammation (fever, leukocyte, CRP, ferritin, etc) must be taken into consideration as well as signs of hypoxia, respiratory failure, shock, and multiple organ failure in evaluating the effectiveness of treatment.

\section{CONCLUSION}

As a result; COVID-19 is a systemic infection that has an important effect on hemostasis and currently has no effective treatment. Multiple organ failure with severe pneumonia, ARDS, sepsis, septic shock, myocarditis, cardiogenic shock can be encountered. Respiratory failure is often in the form of hypoxemic respiratory failure. Management of patients with COVID-19 requires patient isolation, infection prevention and control, optimized supportive care, symptomatic therapy and respiratory support. The prognosis of patients depends on the severity of the disease, the patient's age, underlying diseases, and general medical condition. Patients with severe COVID-19 should be treated in the ICU.

\section{Ethics}

Peer-review: Externally peer-reviewed.

\section{Authorship Contributions}

Concept: S..V., E.A.T., F.G., F.U., T.M., O.O., C.P., N.T., Design: S.V., E.A.T., T.M., O.O., C.P., N.T., Data Collection or Processing: S.V., E.A.T., N.T., Analysis or Interpretation: S.V., N.T., Literature Search: S..V., E.A.T., F.G., F.U., T.M., C.P., N.T., Writing: S.V., E.A.T., F.G., F.U., T.M., O.O., C.P., N.T.

Conflict of Interest: No conflict of interest was declared by the authors.
Financial Disclosure: The authors declared that this study received no financial support. Şule

\section{REFERENCES}

1. T.C. Sağlık Bakanlığı Halk Sağlığı Genel Müdürlüğü COVID-19 (SARSCoV-2 Enfeksiyon Rehberi 14.04.2020 Available from: URL: https://www. tjod.org/wp-content/uploads/2020/04/COVID-19_Rehberi-14.04.2020. pdf

2. Liu J, Liu S. The management of coronavirus disease 2019 (COVID-19). J Med Virol 2020:92:1484-90.

3. Goh KJ, Choong MC, Cheong EH, Kalimuddin S, Duu Wen S, Phua GC, et al. Rapid Progression to Acute Respiratory Distress Syndrome: Review of Current Understanding of Critical IIIness from COVID-19 Infection. Ann Acad Med Singap 2020;49:108-18.

4. Wujtewicz M, Dylczyk-Sommer A, Aszkiełowicz A, Zdanowski S, Piwowarczyk S, Owczuk R. COVID-19 - what should anaethesiologists and intensivists know about it? Anaesthesiol Intensive Ther 2020;52:3441.

5. Rodriguez-Morales AJ, Cardona-Ospina JA, Gutiérrez-Ocampo E, Villamizar-Peña R, Holguin-Rivera Y, Escalera-Antezana JP, et al. Clinical, laboratory and imaging features of COVID-19: A systematic review and meta-analysis. Travel Med Infect Dis 2020;34:101623.

6. Wu Z, McGoogan JM. Characteristics of and Important Lessons From the Coronavirus Disease 2019 (COVID-19) Outbreak in China: Summary of a Report of 72314 Cases From the Chinese Center for Disease Control and Prevention. JAMA 2020;323:1239-42.

7. Wang D, Hu B, Hu C, Zhu F, Liu X, Zhang J, et al. Clinical characteristics of 138 hospitalized patients with 2019 novel coronavirus-infected pneumonia in Wuhan, China. JAMA 2020;323:1061-9.

8. Wilcox SR. Management of respiratory failure due to covid-19. BMJ 2020;369:m1786.

9. Gattinoni L, Chiumello D, Rossi S. COVID-19 pneumonia: ARDS or not? Crit Care 2020;24:154.

10. Alhazzani W, Møller MH, Arabi YM, Loeb M, Gong MN, Fan E, et al Surviving Sepsis Campaign: Guidelines on the Management of Critically III Adults With Coronavirus Disease 2019 (COVID-19) Intensive Care Med 2020;46:854-87.

11. Xu X, Han M, Li T, Sun W, Wang D, Fu B, et al. Effective treatment of severe COVID-19 patients with tocilizumab. Proc Natl Acad Sci USA 2020;117:10970-5. 


\section{Appendix 1. Endotracheal intubation protocol (10)}

1. It should be applied with a rapid sequential intubation protocol by trained and experienced people.

2. Intubation should be performed with a video laryngoscope, if possible. Patients who are thought to have difficult airway can be intubated by flexible bronchoscopy. However, bronchoscopy is also a procedure with a high risk of creating aerosol.

3. Intubation should be applied with maximum personal protective equipment (PPE) in rooms with negative pressure if possible due to the risk of creating aerosol, and if not in single rooms.

4. If possible, the use of balloon-mask should be avoided during preoxygenation. A filter should also be used in balloon mask application.

5. A neuromuscular blocker can be used to suppress cough before intubation. Positive pressure ventilation should not be initiated until the endotracheal cuff is inflated. A heat-moisture exchanger (humidifier) filter can be used, but active humidification should be preferred in cases of dense plugging and dead space increase.

6. Unless necessary, the connection should not be cut in the mechanical ventilator circuit, if it is necessary, PPE must be used.

7. If possible, closed system aspiration method should be used.

8. Bronchoscopic procedures should be avoided unless absolutely necessary, and metered dose inhaler should be preferred instead of nebulization in bronchodilator therapy.

\section{Appendix 2. Tracheostomy protocol in intensive care unit in our hospital (1)}

- Patients who will undergo tracheostomy should have mechanical ventilation values of $\mathrm{FiO}_{2} \leq 50 \%$ and positive end-expiratory pressure $\leq 10$ $\mathrm{mmHg}$.

- In the case of prolonged intubation, daily endotracheal tube cuff pressure measurement is recommended until the tracheostomy is opened. The cuff pressure should be kept in the range of $20-30 \mathrm{~cm} \mathrm{H}_{2} \mathrm{O}$ to prevent air leaks.

- Tracheostomy cannula should be fenestrated and a cuffed cannula. It is useful to have several sizes of tracheostomy cannula ready. In addition, the integrity of the cuff should be checked before the procedure.

- There should be a minimum of healthcare workers in the operation room or the patient's room and all necessary materials should be prepared in the room before.

- Before application, $\mathrm{FiO}_{2}$ should be adjusted to $100 \%$.

- A full dose of neuromuscular block and deep sedation should be applied in the patient at a level to prevent coughing and straining.

- During the opening of the tracheostomy, the use of electrocautery or aspirator should be avoided as much as possible, as they create aerosol.

- During tracheostomy, the cuff of the tube should be fully inflated and adjusted to avoid leakage, and care should be taken not to damage the cuff during surgery.

- When the front wall of the trachea is seen, cutting the cuff should be prevented by pushing forward the anesthesia tube as deep as possible towards the bronchi. Make sure that the cuff of the tube is fully inflated. The surgeon should make the incision and open the tracheal window without damaging the head.

- The most risky time in tracheostomy is the stage of pulling the endotracheal tube and placing the tracheostomy cannula. During this period, if the patient's condition allows (no critical hypoxia), ventilation is stopped at the end of expiration. The endotracheal tube is pulled up to the incision, but is not completely removed, the tube is clamped at this stage.

- Heat and moisture exchanger filter and mechanical ventilation circuit are placed on the tracheostomy cannula. As soon as the cannula is placed, the cuff must be inflated before ventilation is initiated and then the patient must be ventilated. The endotracheal tube should be pulled out and placed in a plastic bag for disposal.

- The location of the tracheal cannula should be verified with end-tidal $\mathrm{CO}_{2}$ measurement, and verification by auscultation with a stethoscope is not recommended in terms of spread of infection.

- If a percutaneous tracheostomy method is used, it can be applied without using bronchospy to minimize aerosol generation. In addition, if the patient's condition is suitable (if there is no critical hypoxia) after the guidwire is placed, mechanical ventilation should be stopped at the end of expiration and the operation area should be covered with gauze before starting tracheal dilatation. 


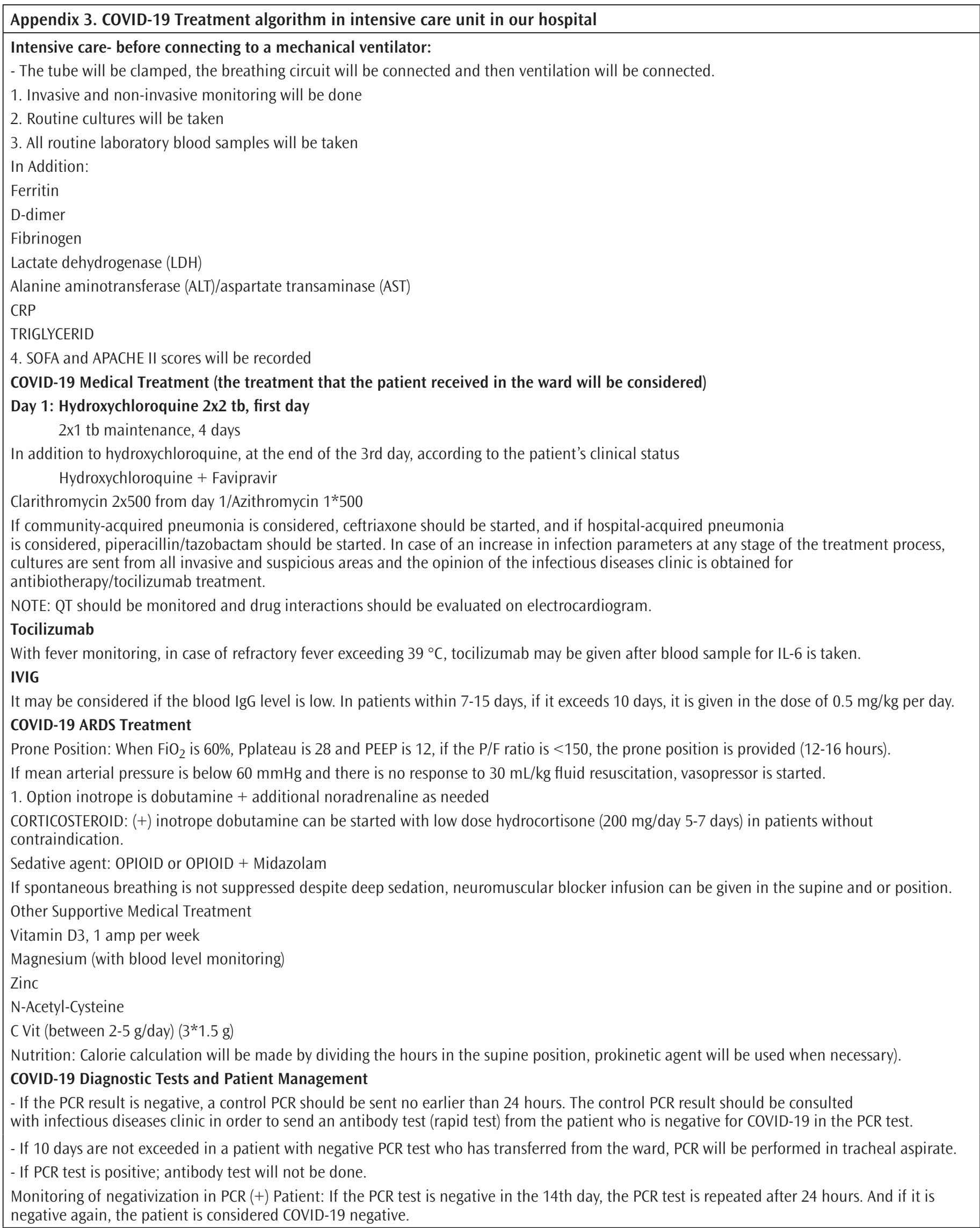

